\title{
FEATURE SELECTION IN SVM VIA POLYHEDRAL $K$-NORM
}

\author{
M. GAUDIOSO*, E. GORGONE ${ }^{\dagger}$, AND J.-B. HIRIART-URRUTY
}

\begin{abstract}
We treat the Feature Selection problem in the Support Vector Machine (SVM) framework by adopting an optimization model based on use of the $\ell_{0}$ pseudo-norm. The objective is to control the number of non-zero components of normal vector to the separating hyperplane, while maintaining satisfactory classification accuracy. In our model the polyhedral norm $\|\cdot\|_{[k]}$, intermediate between $\|\cdot\|_{1}$ and $\|\cdot\|_{\infty}$, plays a significant role, allowing us to come out with a DC (Difference of Convex) optimization problem that is tackled by means of DCA algorithm.

The results of several numerical experiments on benchmark classification datasets are reported.
\end{abstract}

Keywords. Sparse optimization, Cardinality constraint, $k$-norm, Support Vector Machine, DC optimization.

1. Introduction. A relevant problem in binary classification is to design good quality classifiers by resorting to a minimal number of sample parameters. One of the main motivations is to gather a more clear interpretation of phenomena underlying the class membership distribution of the samples. In the more general setting of Machine Learning, such problem falls in the area of Feature Selection (FS), which has been the object of intensive research in recent decades (see, e.g., the survey [10]).

We focus, in particular, on the Support Vector Machine (SVM) framework [24], [4], where binary classification is pursued by finding an "optimal" two-class separating hyperplane, either in the original parameter (or "feature") space or upon appropriate kernel transformation.

Numerical optimization algorithms play a relevant role in SVM area and, more specifically, in FS. The problem is to guarantee a reasonable trade-off between classification accuracy and the number of features actually used. Controlling the latter consists basically in minimizing the number of non-zero components of the normal vector to the separating hyperplane.

The literature offers several contributions. In [1] NP-hardness of the problem has been assessed.

In [3] the model adopted is based on considering the step function for each component of the normal vector; discontinuity is handled by introducing two different approximations, the standard sigmoid and a concave exponential, respectively. In particular, by adopting the concave approximation, FS problem is tackled by solving a finite sequence of linear programs. Different approximations are given in [26] and in [21], where, in particular, the concave and separable objective functions, derived by the approximations, are handled via variants of Frank-Wolfe method.

It is interesting to note that in [16] an approximation scheme of the step function is cast into a DC (Difference of Convex) framework, providing thus the opportunity of resorting to the algorithmic machinery for dealing with such class of nonconvex problems. An early survey on properties and relevance of such class of functions is in [12] (see also [22]).

Parallel to treatment of FS via approximation methods, Mixed Integer Programming formulations have been successfully adopted. The idea is to introduce binary variables, one for each component of the normal vector to the supporting hyperplane, that are switched to " 1 " if and only if the corresponding component is non-zero. From among the proposed approaches, we recall here [17], [2], [7].

In a more general setting, FS falls into the wide area of sparse optimization, where one is faced to

*Dipartimento di Ingegneria Informatica, Modellistica, Elettronica e Sistemistica, Università della Calabria, 87036 Rende (CS), Italia. E-mail: gaudioso@dimes.unical.it

†Dipartimento di Matematica e Informatica, Università di Cagliari, 09124 Cagliari, Italia. E-mail: egorgone@unica.it

¥Institut de mathématiques, Université Paul Sabatier, 31062 Toulouse, France. E-mail: jbhu@math.univ-toulouse.fr 
the (regularized) problem:

$$
\min _{x \in \mathbb{R}^{n}} f(x)+\|x\|_{0}
$$

where $f: \mathbb{R}^{n} \rightarrow \mathbb{R}$ is convex and $\|\cdot\|_{0}$ is the $\ell_{0}$ pseudo-norm, which counts the number of non-zero component of any vector. Sometimes sparsity of the solution, instead of acting on the objective function, is enforced by introducing a constraint on the $\ell_{0}$ pseudo-norm of the solution, thus defining a cardinalityconstraint problem [20].

In many applications, the $\ell_{0}$ pseudo-norm in (1.1) is replaced by the $\ell_{1}-$ norm, which is definitely more tractable from the computational point of view, yet ensuring sparsity, to a certain extent (see [27] for a discussion on a general regularization scheme).

In the seminal paper [25], a class of polyhedral norms (the $k$-norms), intermediate between $\|\cdot\|_{1}$ and $\|\cdot\|_{\infty}$, is introduced to obtain sparse approximation solutions to systems of linear equations. The use of other norms to recover sparsity is described in [6]. In more recent years the use of $k$-norms has received much attention and has led to several proposals for dealing with $\ell_{0}$ pseudo-norm cardinality constrained problem [11], [28], [23], [9].

In this paper we cast the classic SVM approach into the sparse optimization framework (1.1). Our work is inspired by [9], the main difference being in the explicit (and not parametric) minimization of the $\ell_{0}$ pseudo-norm. We formulate our SVM- $\ell_{0}$ pseudo-norm problem $\left(S V M_{0}\right.$, for short) and we tackle it by means of a penalization approach which allows us to put the problem in DC form. The algorithm adopted is of DCA type [15].

The paper is organized as follows. In Section 2 we recall first the standard SVM model and introduce the FS problem as a sparse optimization one. Then we discuss the use of the polyhedral $k$-norm in sparse optimization, coming out with a DC formulation. On such basis we formulate in Section 3 the $S V M_{0}$ problem. The results of several numerical experiments on benchmark datasets are in Section 4. Some conclusions are finally drawn in Section 5.

2. Feature Selection and $\ell_{0}$ pseudo-norm minimization. The binary classification problem in the SVM setting is usually put in the following form. Given two point-sets $\mathcal{A} \triangleq\left\{a_{1}, \ldots, a_{m_{1}}\right\}$ and $\mathcal{B} \triangleq\left\{b_{1}, \ldots, b_{m_{2}}\right\}$ in $\mathbb{R}^{n}$, we look for linear separation of the two sets, that is for a hyperplane $\{x \mid x \in$ $\left.\mathbb{R}^{n}, x^{\top} w^{\prime}=\gamma^{\prime}\right\},\left(w^{\prime} \in \mathbb{R}^{n}, \gamma^{\prime} \in \mathbb{R}\right)$, strictly separating $\mathcal{A}$ and $\mathcal{B}$, thus ensuring

$$
a_{i}^{\top} w^{\prime}<\gamma^{\prime}, \quad i=1, \ldots, m_{1} \text { and } b_{l}^{\top} w^{\prime}>\gamma^{\prime}, \quad l=1, \ldots, m_{2} .
$$

It is easy to verify that such a hyperplane exists if and only if there exists a hyperplane $\{x \mid x \in$ $\left.\mathbb{R}^{n}, x^{\top} w=\gamma\right\},\left(w \in \mathbb{R}^{n}, \gamma \in \mathbb{R}\right)$, such that

$$
a_{i}^{\top} w \leq \gamma-1, \quad i=1, \ldots, m_{1} \text { and } b_{l}^{\top} w \geq \gamma+1, \quad l=1, \ldots, m_{2} .
$$

Gordan's theorem of the alternative [18] guarantees linear separation if and only if $\operatorname{conv} \mathcal{A} \cap \operatorname{conv} \mathcal{B}=\emptyset$, a property which is not usually known in advance to hold.

Consequently, an error function of $(w, \gamma)$, which is convex, piecewise linear and nonnegative, is introduced. It assumes zero value if and only if $(w, \gamma)$ actually defines a (strictly) separating hyperplane and it has the form:

$$
e(w, \gamma)=\sum_{i=1}^{m_{1}} \max \left\{0, a_{i}^{\top} w-\gamma+1\right\}+\sum_{l=1}^{m_{2}} \max \left\{0,-b_{l}^{\top} w+\gamma+1\right\}
$$


The SVM approach consists in solving the following convex problem:

$$
\min _{w, \gamma}\|w\|+C e(w, \gamma)
$$

where the addition of the norm of $w$ to the error function is aimed at obtaining a maximum-margin separation, $C$ being a positive trade-off parameter [4].

In the standard approach $\ell_{1}$ or $\ell_{2}$ norms are usually adopted in the definition of problem (2.2), while for feature selection purposes the $\ell_{0}$ pseudo-norm, which counts the number of non-zero components of any vector, is introduced.

The usual notation $\|\cdot\|_{0}$ for indicating the $\ell_{0}$ pseudo-norm is motivated by the observation

$$
\left(\|\cdot\|_{p}\right)^{p} \rightarrow\|\cdot\|_{0} \quad \text { when } \quad p \rightarrow 0 .
$$

Relevant properties of function $x \mapsto\|x\|_{0}$ are:

i) it is lower-semicontinuous, that is to say

$$
\lim \inf _{k \rightarrow+\infty}\left\|x_{k}\right\|_{0} \geq\|x\|_{0} \quad \text { whenever } \quad x_{k} \rightarrow x
$$

a property which is fundamental in view of using descent algorithms;

ii) it is homogeneous of degree $0,\left(\|\lambda x\|_{0}=\|x\|_{0}\right.$, for $\left.\lambda \neq 0\right)$; constancy along rays makes difficult the design of minimization algorithms;

iii) the convex hull of $\|\cdot\|_{0}$ on the ball $\left\{x \mid\|x\|_{\infty} \leq r\right\}$ is exactly the function $\frac{1}{r}\|\|_{1}$. This is a mathematical justification for frequent substitution of $\|\cdot\|_{0}$ by $\|\cdot\|_{1}$, which in fact ensures, in practical applications, attractive sparsity properties of the solution.

In our approach we fix $\|w\|_{0}$ in formulation (2.2), thus we consider a sparse optimization problem of the type

$$
f_{0}^{*}=\min _{x \in \mathbb{R}^{n}} f(x)+\|x\|_{0},
$$

where $f: \mathbb{R}^{n} \rightarrow \mathbb{R}, n \geq 2$, is convex, not necessarily differentiable. We observe, in passing, the significant parallelism between sparse optimization and certain problems in matrix optimization [19], [13]. See, in particular, the recent approach to the rank function minimization described in [9].

In the sequel, we resort to the use of $\|x\|_{[k]}$, the vector $k$-norm of $x$, which is defined as the sum of $k$ largest components (in modulus) of $x, k=1, \ldots, n$. In fact $\|\cdot\|_{[k]}$ is a polyhedral norm, intermediate between $\|\cdot\|_{1}$ and $\|\cdot\|_{\infty}$.

The following properties hold:

i) $\|x\|_{\infty}=\|x\|_{[1]} \leq \ldots \leq\|x\|_{[k]} \leq \ldots\|x\|_{[n]}=\|x\|_{1}$;

ii) $\|x\|_{0} \leq k \Rightarrow\|x\|_{1}-\|x\|_{[s]}=0, \quad k \leq s \leq n$.

Moreover, it is easy to prove the equivalence, valid for $1 \leq k \leq n$,

$$
\|x\|_{0} \leq k \Leftrightarrow\|x\|_{1}-\|x\|_{[k]}=0,
$$

which allows to replace any constraint of the type $\|x\|_{0} \leq k$ with a difference of norms, that is a DC constraint.

Taking any point $\bar{x} \in \mathbb{R}^{n}$ and letting $I_{[k]} \triangleq\left\{i_{1}, \ldots, i_{k}\right\}$ be the index set of $k$ largest in modulus components of $\bar{x}$, a subgradient $\bar{g}^{[k]}$ of the vector $k$-norm at $\bar{x}$ can be calculated by setting:

$$
\bar{g}_{i}^{[k]}=\left\{\begin{array}{lll}
1 & \text { if } & i \in I_{[k]} \text { and } \bar{x}_{i} \geq 0 \\
-1 & \text { if } & i \in I_{[k]} \text { and } \bar{x}_{i}<0 \\
0 & \text { otherwise }
\end{array}\right.
$$


To tackle problem 2.3, we start from the observation (see [19], [28]):

$$
\|x\|_{[k]}=\max _{y \in \psi_{k}} y^{\top} x
$$

where $\psi_{k}$ is the subdifferential of $\|\cdot\|_{0}$ at point 0 ,

$$
\psi_{k}=\left\{y \in \mathbb{R}^{n} \mid y=u-v, 0 \leq u, v \leq e,(u+v)^{\top} e=k\right\},
$$

with $e$ being the vector of $n$ ones. Then we formulate the following problem:

$$
\begin{aligned}
f_{c}^{*}= & \min _{x, u, v, z} f(x)+z \\
& e^{\top}(u+v)=z \\
& (u-v)^{\top} x \geq\|x\|_{1} \\
& 0 \leq u, v \leq e, \quad x \in \mathbb{R}^{n} .
\end{aligned}
$$

REMARK 2.1. At any feasible point constraint (2.7) is satisfied by equality.

Proposition 2.2. At any optimal (local) solution $\left(x^{*}, u^{*}, v^{*}, z^{*}\right)$ of problem 2.5-2.8, the following relations hold for $j=1, \ldots, d$ :

$$
\begin{cases}x_{j}^{*}>0 & \Leftrightarrow u_{j}^{*}=1 \\ x_{j}^{*}<0 & \Leftrightarrow \quad v_{j}^{*}=1 \\ x_{j}^{*}=0 & \Leftrightarrow \quad u_{j}^{*}=v_{j}^{*}=0\end{cases}
$$

Proof. Note first that $u_{j}^{*}$ and $v_{j}^{*}$ cannot be both positive. In fact, in such case the solution obtained by replacing $u_{j}^{*}$ and $v_{j}^{*}$ by $u_{j}^{*}-\delta_{j}$ and $v_{j}^{*}-\delta_{j}$, with $\delta_{j} \triangleq \min \left\{u_{j}^{*}, v_{j}^{*}\right\}>0$, would be still feasible and would reduce the objective function value.

Now observe that, while constraint 2.8 ensures

$$
-\left|x_{j}\right| \leq x_{j}\left(u_{j}-v_{j}\right) \leq\left|x_{j}\right|, \quad j=1, \ldots, d,
$$

satisfaction of constraint 2.7 guarantees $x_{j}\left(u_{j}-v_{j}\right)=\left|x_{j}\right|, j=1, \ldots, d$. Thus it is proved, in particular, $x_{j}^{*}>0 \Rightarrow u_{j}^{*}=1\left(x_{j}^{*}<0 \Rightarrow v_{j}^{*}=1\right)$.

The implication $x_{j}^{*}=0 \Rightarrow u_{j}^{*}=v_{j}^{*}=0$ can be proved by a simple contradiction argument, taking into account optimality of the solution. The same contradiction argument guarantees that the implications $x_{j}^{*}>0 \Leftarrow u_{j}^{*}=1\left(x_{j}^{*}<0 \Leftarrow v_{j}^{*}=1\right)$ hold true, while the last implication $x_{j}^{*}=0 \Leftarrow u_{j}^{*}=v_{j}^{*}=0$ can be proved by observing that $u_{j}^{*}=v_{j}^{*}=0$ and $x_{j}^{*} \neq 0$ would lead to violation of constraint 2.7.

REMARK 2.3. Implications 2.9 ensure $z^{*}=\left\|x^{*}\right\|_{0}$. Moreover, letting $\left(x^{*}, u^{*}, v^{*}, z^{*}\right)$ be any local minimum of problem 2.5-2.8, then $x^{*}$ is a local minimum for problem 2.3.

By eliminating the scalar variable $z$ in problem 2.5-2.8 we come out with the reformulation

$$
\begin{aligned}
f_{c}^{*}= & \min _{x, u, v} f(x)+e^{\top}(u+v) \\
& (u-v)^{\top} x \geq\|x\|_{1} \\
& 0 \leq u, v \leq e, \quad x \in \mathbb{R}^{n},
\end{aligned}
$$


which we approach by penalizing the nonlinear nonconvex constraint 2.11 through the scalar penalty parameter $\sigma>0$. We obtain

$$
\begin{gathered}
f_{c}(\sigma)=\min _{x, u, v} f(x)+e^{\top}(u+v)+\sigma\left(\|x\|_{1}-(u-v)^{\top} x\right) \\
0 \leq u, v \leq e, \quad x \in \mathbb{R}^{n} .
\end{gathered}
$$

The objective function 2.13 is suitable for a DC (Difference of Convex) decomposition.

Observe, in fact, that, letting $p^{\top} \triangleq\left(x^{\top}, u^{\top}, v^{\top}\right)$, the function

$$
q(p) \triangleq(v-u)^{\top} x,
$$

can be written in DC form as

$$
q(p)=p^{\top} Q_{1} p-p^{\top} Q_{2} p,
$$

where the symmetric positive semidefinite matrices $Q_{1}$ and $Q_{2}$ of dimension $(3 n, 3 n)$ are defined as follows:

$$
Q_{1}=\frac{1}{4}\left(\begin{array}{rrr}
2 I & -I & I \\
-I & I & 0 \\
I & 0 & I
\end{array}\right)
$$

and

$$
Q_{2}=\frac{1}{4}\left(\begin{array}{rrr}
2 I & I & -I \\
I & I & 0 \\
-I & 0 & I
\end{array}\right),
$$

with $I$ and 0 being, respectively, the identity matrix and the zero matrix of dimension $(n, n)$.

Summing up, the objective function of problem 2.13-2.14 is decomposed as follows:

$$
f(x)+e^{\top}(u+v)+\sigma\left(\|x\|_{1}-(u-v)^{\top} x\right)=h_{1}(p)-h_{2}(p),
$$

with

$$
h_{1}(p) \triangleq f(x)+e^{\top}(u+v)+\sigma\left(\|x\|_{1}+p^{\top} Q_{1} p\right),
$$

and

$$
h_{2}(p) \triangleq \sigma p^{\top} Q_{2} p .
$$

Before concluding the Section, we state a property related to parametric normalization of a convex function via the $\ell_{1}$ norm. It will be useful in explaining the role of parameter $\sigma$ in the numerical experiments of Section 4.

Proposition 2.4. Let $f: \mathbb{R}^{n} \rightarrow \mathbb{R}$ be convex and define, for $\sigma>0, f_{\sigma}(x)=f(x)+\sigma\|x\|_{1}$. If

$$
\sigma>\min _{g \in \partial f(0)}\|g\|_{\infty}
$$


then $x^{*}=0$ is the unique minimum of $f_{\sigma}$.

Proof. Our aim is to prove

$$
f(x)+\sigma\|x\|_{1}>f(0) \quad \forall x \in \mathbb{R}^{n}, \quad x \neq 0 .
$$

According to the definition of $g \in \partial f(0)$,

$$
f(x) \geq f(0)+g^{\top} x, \quad \forall x \in \mathbb{R}^{n},
$$

whence

$$
f(x)+\sigma\|x\|_{1} \geq f(0)+\sigma\|x\|_{1}+g^{\top} x, \quad \forall x \in \mathbb{R}^{n} .
$$

Since $g^{\top} x \geq-\|g\|_{\infty}\|x\|_{1}$, we infer from the above

$$
f(x)+\sigma\|x\|_{1} \geq f(0)+\left(\sigma-\|g\|_{\infty}\right)\|x\|_{1}, \quad \forall x \in \mathbb{R}^{n} .
$$

To ensure (2.17), it suffices fo have $\sigma-\|g\|_{\infty}>0$ for one $g \in \partial f(0)$. This is secured with the assumption $\sigma>\min _{g \in \partial f(0)}\|g\|_{\infty}$.

$\square$

3. The $S V M_{0}$ problem. We rewrite first the SVM model (2.2), taking into account the definition (2.1) of the classification error and adopting the $\ell_{1}$ norm. We obtain problem $S V M_{1}$.

$$
z^{*}=\min _{w, \gamma, \xi, \zeta}\|w\|_{1}+C\left(\sum_{i=1}^{m_{1}} \xi_{i}+\sum_{l=1}^{m_{2}} \zeta_{l}\right)
$$

subject to

$$
\begin{array}{lc}
a_{i}^{\top} w-\gamma+1 \leq \xi_{i}, & i=1, \ldots, m_{1} \\
-b_{l}^{\top} w+\gamma+1 \leq \zeta_{l}, & l=1, \ldots, m_{2} \\
\xi_{i} \geq 0, & i=1, \ldots, m_{1} \\
\zeta_{l} \geq 0, & l=1, \ldots, m_{2},
\end{array}
$$

where the nonnegative auxiliary variables $\xi_{i}, i=1, \ldots, m_{1}$ and $\zeta_{l}, l=1, \ldots, m_{2}$ have been introduced to eliminate nonsmoothness in the definition (2.1).

Moreover, by letting

$$
w=w^{+}-w^{-}, w^{+}, w^{-} \geq 0,
$$

and indicating by $e$ the vector of ones of dimension $n$, the above problem can be rewritten in a Linear 
Programming form as follows

$$
z^{*}=\min _{w^{+}, w^{-}, \gamma, \xi, \zeta} e^{\top}\left(w^{+}+w^{-}\right)+C\left(\sum_{i=1}^{m_{1}} \xi_{i}+\sum_{l=1}^{m_{2}} \zeta_{l}\right)
$$

subject to

$$
\begin{array}{lc}
a_{i}^{\top}\left(w^{+}-w^{-}\right)-\gamma+1 \leq \xi_{i}, & i=1, \ldots, m_{1} \\
-b_{l}^{\top}\left(w^{+}-w^{-}\right)+\gamma+1 \leq \zeta_{l}, & l=1, \ldots, m_{2} \\
\xi_{i} \geq 0, & i=1, \ldots, m_{1} \\
\zeta_{l} \geq 0, & l=1, \ldots, m_{2}, \\
w^{+} \geq 0, w^{-} \geq 0 &
\end{array}
$$

Of course 3.1-3.5 or 3.6-3.11 are equivalent formulations of $S V M_{1}$.

We remark that choice of $\|\cdot\|_{1}$ in $(2.2)$, instead of $\|\cdot\|_{2}$, has a beneficial effect in terms of feature selection (see [3]).

To guarantee, however, a better control on the number of features actually entering the classification process, we replace $\|\cdot\|_{1}$ with $\|.\|_{0}$ and adapt to SVM the sparse optimization approach described in the previous section. We obtain the $S V M_{0}$ problem

$$
z^{*}=\min _{w^{+}, w^{-}, \gamma, \xi, \zeta, u, v} C\left(\sum_{i=1}^{m_{1}} \xi_{i}+\sum_{l=1}^{m_{2}} \zeta_{l}\right)+e^{\top}(u+v)
$$

subject to

$$
\begin{aligned}
& (u-v)^{\top}\left(w^{+}-w^{-}\right) \geq e^{\top}\left(w^{+}+w^{-}\right) \\
& a_{i}^{\top}\left(w^{+}-w^{-}\right)-\gamma+1 \leq \xi_{i}, \\
& -b_{l}^{\top}\left(w^{+}-w^{-}\right)+\gamma+1 \leq \zeta_{l}, \\
& \xi_{i} \geq 0, \\
& \zeta_{l} \geq 0, \\
& w^{+} \geq 0, w^{-} \geq 0 \\
& 0 \leq u, v \leq e
\end{aligned}
$$


Penalizing the (nonlinear) constraint (3.13) we obtain

$$
z^{*}=\min _{w^{+}, w^{-}, \gamma, \xi, \zeta, u, v} e^{\top}(u+v)+C\left(\sum_{i=1}^{m_{1}} \xi_{i}+\sum_{l=1}^{m_{2}} \zeta_{l}\right)+\sigma\left(e^{\top}\left(w^{+}+w^{-}\right)-(u-v)^{\top}\left(w^{+}-w^{-}\right)\right)
$$

subject to

$$
\begin{aligned}
& a_{i}^{\top}\left(w^{+}-w^{-}\right)-\gamma+1 \leq \xi_{i}, \quad i=1, \ldots, m_{1} \\
& -b_{l}^{\top}\left(w^{+}-w^{-}\right)+\gamma+1 \leq \zeta_{l}, l=1, \ldots, m_{2} \\
& \xi_{i} \geq 0, \quad i=1, \ldots, m_{1} \\
& \zeta_{l} \geq 0, \quad l=1, \ldots, m_{2}, \\
& w^{+} \geq 0, \quad w^{-} \geq 0 \\
& 0 \leq u, v \leq e
\end{aligned}
$$

REMARK 3.1. Definition of an exact nondifferentiable penalty function of the type described in [5] would require the introduction into the objective function of the term

$$
\sigma \max \left(0, e^{\top}\left(w^{+}+w^{-}\right)-(u-v)^{\top}\left(w^{+}-w^{-}\right)\right),
$$

which in our case, according to remark (2.1), is simply replaced by

$$
\sigma\left(e^{\top}\left(w^{+}+w^{-}\right)-(u-v)^{\top}\left(w^{+}-w^{-}\right)\right),
$$

giving rise to a differentiable exact penalty function

Problem (3.20)-(3.26) can be put in DC form. In fact, letting $s^{\top} \triangleq\left(w^{+T}, w^{-T}, u^{\top}, v^{\top}\right)$, the function

$$
r(s) \triangleq(v-u)^{\top}\left(w^{+}-w^{-}\right)
$$

can be rewritten as

$$
r(s)=s^{\top} \hat{Q}_{1} s-s^{\top} \hat{Q}_{2} s,
$$

where the symmetric positive semidefinite matrices $\hat{Q}_{1}$ and $\hat{Q}_{1}$ of dimension $(4 d, 4 d)$ are defined as follows

$$
\hat{Q}_{1}=\frac{1}{4}\left(\begin{array}{rrrr}
2 I & -2 I & -I & I \\
-2 I & +2 I & I & -I \\
-I & I & I & 0 \\
I & -I & 0 & I
\end{array}\right)
$$

and

$$
\hat{Q}_{2}=\frac{1}{4}\left(\begin{array}{rrrr}
2 I & -2 I & I & -I \\
-2 I & +2 I & -I & I \\
I & -I & I & 0 \\
-I & I & 0 & I
\end{array}\right)
$$


with $I$ and 0 being, respectively, the identity and the zero matrix of dimension $(d, d)$.

The objective function is then decomposed in DC form $f_{1}\left(w^{+}, w^{-}, \gamma, \xi, \zeta, u, v\right)-f_{2}\left(w^{+}, w^{-}, u, v\right)$ with

$$
f_{1}\left(w^{+}, w^{-}, \gamma, \xi, \zeta, u, v\right)=e^{\top}(u+v)+C\left(\sum_{i=1}^{m_{1}} \xi_{i}+\sum_{l=1}^{m_{2}} \zeta_{l}\right)+\sigma\left(e^{\top}\left(w^{+}+w^{-}\right)+s^{\top} \hat{Q}_{1} s\right),
$$

and

$$
f_{2}\left(w^{+}, w^{-}, u, v\right)=\sigma s^{\top} \hat{Q}_{1} s
$$

We apply to problem above the DCA method [15], which tackles the unconstrained minimization of a function $q: \mathbb{R}^{n} \rightarrow \mathbb{R}$

$$
q(x)=q_{1}(x)-q_{2}(x)
$$

with $q_{1}$ and $q_{2}$ convex, by solving a sequence of linearized convex problems. In particular, letting $x^{(k)}$ be the estimate of a (local) minimum of $g$ at iteration $k$, the next iterate $x^{(k+1)}$ is calculated as

$$
x^{(k+1)}=\arg \min _{x \in \mathbb{R}^{n}}\left\{q_{1}(x)-\left[q_{2}\left(x^{(k)}\right)+g^{(k) \top}\left(x-x^{(k)}\right)\right]\right\},
$$

with $g^{(k)} \in \partial q_{2}\left(x^{(k)}\right)$. For other methods to solve DC problems see [14], [8] and the references therein.

We remark that, in applying the successive linearization method to our case, a convex quadratic minimization problem is solved at each iteration.

4. Numerical experiments. We have performed our experiments on two groups of five datasets each. They are the same datasets adopted as benchmark for the feature selection method described in [7]. In particular in datasets 1-5 (Group 1), available at http://www.tech.plym.ac.uk/spmc/, the number of samples is small with respect to the number of features. The opposite happens for Datasets 610 (Group 2), which are available at https://www.csie.ntu.edu.tw/ cjlin/libsvmtools/datasets/. Thus for the latter ones a certain class-overlap is expected.

The datasets are listed in Table 4.1, where $m=m_{1}+m_{2}$ is the total number of samples.

\begin{tabular}{|r|rr|r|rr|}
\hline Datasets of Group 1 & $m$ & $n$ & Datasets of Group 2 & $m$ & $n$ \\
\hline Carcinoma (CARC) & 36 & 7457 & Breast Cancer (BC) & 683 & 10 \\
DLBCL & 77 & 7129 & PIMA Indians Diabetes (PIMA) & 768 & 8 \\
Leukemia (LEK) & 72 & 5327 & HEART & 270 & 13 \\
Tumor1 (TUM1) & 60 & 7129 & Ionosphere (IONO) & 351 & 34 \\
Tumor2 (TUM2) & 50 & 12625 & Liver Disorders (LIVER) & 145 & 5 \\
\hline \multicolumn{7}{c}{ Tescription of the datasets }
\end{tabular}

As a possible reference, we report first the results of the $S V M_{1}$ problem provided by CPLEX solver. A standard tenfold cross validation has been performed. The results are in Table 4.2, where the columns "Test" and "Train" indicate the average testing and training correctness, respectively, expressed as percentage of samples correctly classified. Columns " $\|w\|_{1}$ " and "Time" report the average $\ell_{1}$ norm of $w$ and the average execution time (in seconds). Finally columns "\% $\mathrm{ft}(0)$ " - "\% $\mathrm{ft}(-9)$ " report the average percentage of components of $w$ whose modulus is greater than or equal to $10^{0}-10^{-9}$, respectively. Note that, assuming, conventionally, to be equal to "zero" any component $w_{j}$ of $w$ such that $\left|w_{j}\right|<10^{-9}$, the percentage of zero-components is $(100-\% f t(-9))$. 


\begin{tabular}{|c|r|r|r|r|r|r|r|r|}
\hline & \multicolumn{10}{|c|}{$C=1$} \\
\hline Dataset & Test & Train & $|w|_{1}$ & Time & $\% \mathrm{ft}(0)$ & $\% \mathrm{ft}(-2)$ & $\% \mathrm{ft}(-4)$ & $\% \mathrm{ft}(-9)$ \\
\hline \hline CARC & 97.50 & 100.00 & 2.63 & 0,27 & 0.00 & 0.26 & 0.28 & 0.28 \\
DLBCL & 94,29 & 100.00 & 5.07 & 0.54 & 0.00 & 0.42 & 0.44 & 0.44 \\
LEUK & 96.67 & 100.00 & 4.45 & 0.37 & 0.00 & 0.54 & 0.58 & 0.58 \\
TUM1 & 76.67 & 100.00 & 7.09 & 0.52 & 0.00 & 0.53 & 0.55 & 0.55 \\
TUM2 & 80.50 & 100.00 & 5.12 & 0.69 & 0.00 & 0.23 & 0.24 & 0.24 \\
\hline \hline \multicolumn{10}{|c|}{$C=10$} \\
\hline BC & 97.24 & 94.00 & 6.01 & 0,01 & 9.00 & 94.00 & 94.00 & 94.00 \\
PIMA & 77.62 & 100.00 & 8.30 & 0,02 & 26.25 & 100.00 & 100.00 & 100.00 \\
HEART & 86.83 & 100.00 & 7.27 & 0,01 & 12.31 & 100.00 & 100.00 & 100.00 \\
IONO & 95.46 & 95.59 & 43.43 & 0,01 & 50.59 & 95.00 & 95.59 & 95.59 \\
LIVER & 74.39 & 100.00 & 5.14 & 0,00 & 48.00 & 100.00 & 100.00 & 100.00 \\
\hline
\end{tabular}

Two different values of parameter $C$, obtained through the so called "Model selection" phase (see [7]), have been adopted for the two dataset groups.

Before reporting the results of the implementation of our algorithm to solve $S V M_{0}$, we illustrate in Tables 4.3 and 4.4 the results provided by the following mixed binary programming problem $M B P[7]$ for feature selection, where $\ell_{0}$ pseudo-norm minimization is pursued by introducing the set of binary variables $y_{j}, j=1, \ldots, n$.

$$
z^{*}=\min _{w, \gamma, \xi, \zeta, y}\|w\|_{1}+C\left(\sum_{i=1}^{m_{1}} \xi_{i}+\sum_{l=1}^{m_{2}} \zeta_{l}\right)+D \sum_{j=1}^{n} y_{j}
$$

subject to

$$
\begin{aligned}
& a_{i}^{\top} w+\gamma+1 \leq \xi_{i}, \\
& -b_{l}^{\top} w-\gamma+1 \leq \zeta_{l}, \\
& -u_{j} y_{j} \leq w_{j} \leq u_{j} y_{j}, \\
& \xi_{i} \geq 0, \\
& \zeta_{l} \geq 0, \\
& y_{j} \in\{0,1\},
\end{aligned}
$$

$$
\begin{gathered}
i=1, \ldots, m_{1} \\
l=1, \ldots, m_{2} \\
j=1, \ldots, n \\
i=1, \ldots, m_{1} \\
l=1, \ldots, m_{2} \\
j=1, \ldots, n,
\end{gathered}
$$

where $u_{j}>0, j=1, \ldots, n$, is a given bound on the modulus of the $j$-th component of $w$ (see [7] for a discussion on setting the $u_{j}$ 's) . The binary variable $y_{j}, j=1, \ldots, n$ is equal to 1 at the optimum if and only if $w_{j} \neq 0, j=1, \ldots, n$. Consequently the term $\sum_{j=1}^{n} y_{j}$ in the objective function represents the $\ell_{0}$ pseudo-norm of $w$. The positive parameter $D$ provides the tradeoff between the $\ell_{0}$ pseudo-norm objective and that of the $S V M_{1}$ problem (3.6)-(3.11).

The results are those obtained by CPLEX, with maximum running time of 1000 seconds. Note that on some test problems, the maximum running time has been achieved with no optimality certification. The results we provide in such cases are related to the best solution found.

Some comments are in order. We observe first that the average computation time for solving the 


\begin{tabular}{|c|r|r|r|r|r|r|r|r|}
\hline & \multicolumn{7}{|c|}{$C=1, D=0.01$} \\
\hline Dataset & Test & Train & $\left.|w|\right|_{1}$ & Time & $\% \mathrm{ft}(0)$ & $\% \mathrm{ft}(-2)$ & $\% \mathrm{ft}(-4)$ & $\% \mathrm{ft}(-9)$ \\
\hline CARC & 92.50 & 100 & 2.65 & 302.07 & 0.00 & 0.16 & 0.16 & 0.16 \\
DLBCL & 92.86 & 100 & 5.11 & 999.98 & 0.00 & 0.28 & 0.28 & 0.28 \\
LEUK & 93.57 & 100 & 4.48 & 999.74 & 0.00 & 0.35 & 0.35 & 0.35 \\
TUM1 & 76.67 & 100 & 7.12 & 998.91 & 0.00 & 0.39 & 0.39 & 0.39 \\
TUM2 & 83.00 & 100 & 5.16 & 999.89 & 0.00 & 0.16 & 0.16 & 0.16 \\
\hline
\end{tabular}

MBP - Cplex implementation - Tenfold cross validation - Group 1

\begin{tabular}{|c|r|r|r|r|r|r|r|r|}
\hline & \multicolumn{10}{|c|}{$C=10, D=10$} \\
\hline Dataset & Test & Train & $|w|_{1}$ & Time & $\% \mathrm{ft}(0)$ & $\% \mathrm{ft}(-2)$ & $\% \mathrm{ft}(-4)$ & $\% \mathrm{ft}(-9)$ \\
\hline BC & 96.42 & 97.15 & 5.73 & 0.06 & 17.00 & 68.00 & 68.00 & 68.00 \\
PIMA & 75.87 & 77.26 & 7.75 & 0.06 & 26.25 & 78.75 & 78.75 & 78.75 \\
HEART & 83.95 & 86.97 & 5.16 & 0.07 & 9.23 & 58.46 & 58.46 & 58.46 \\
IONO & 88.29 & 94.62 & 27.06 & 2.03 & 36.18 & 45.29 & 45.29 & 45.29 \\
LIVER & 71.10 & 74.05 & 4.45 & 0.02 & 52.00 & 68.00 & 68.00 & 68.00 \\
\hline
\end{tabular}

MBP - Cplex implementation - Tenfold cross validation - Group 2

Linear Program $S V M_{1}$ is negligible.

As for classification performance, comparison of Table 4.2 with Tables 4.3 and 4.4 highlights that the use of an explicit feature selection mechanism results in a mild downgrading of the classification correctness. Such phenomenon is compensated by the reduction in the percentage of the numerically significant features (columns" $\mathrm{ft}(0)$ " - $\mathrm{ft}(-9)$ ").

Coming now to our method (referred to, in the sequel, as the "SVM$M_{0}$ Algorithm"), we report in Table 4.5 the results on the two groups of datasets.

We have added the two columns "\%Viol." and " $e^{\top}(u+v)$ ". In particular, column "\%Viol." reports the percentage ratio between the average violation of the relaxed constraint 3.13 and the average norm $\|w\|_{1}$. Column " $e^{\top}(u+v)$ " reports the average value of the scalar product $e^{\top}(u+v)$, which, for small values of the companion parameter "\%Viol.", reasonably approximates $\|w\|_{0} .{ }^{\ddagger}$

\begin{tabular}{|c|c|c|c|c|c|c|c|c|c|c|c|}
\hline & \multicolumn{11}{|c|}{$C=1$} \\
\hline Dataset & $\sigma$ & Test & Train & $|w|_{1}$ & Time & \%Viol. & $e^{\top}(u+v)$ & $\% \mathrm{ft}(0)$ & $\% \mathrm{ft}(-2)$ & $\% \mathrm{ft}(-4)$ & $\% \mathrm{ft}(-9)$ \\
\hline CARC & 4 & 97.50 & 100,00 & 6.90 & 39.40 & 3 & 2.10 & 0.03 & 0.05 & 0.06 & 0.06 \\
\hline DLBCL & 4 & 91.43 & 98.57 & 12.83 & 260.48 & 0.5 & 3.51 & 0.05 & 0.06 & 0.06 & 0.06 \\
\hline LEUK & 5 & 98.33 & 99.83 & 16.02 & 188.70 & 0.0 & 3.70 & 0.07 & 0.07 & 0.07 & 0.07 \\
\hline TUM1* & 5 & 76.67 & 100.00 & 15.27 & 50.74 & 4.7 & 7.23 & 0.08 & 0.14 & 0.16 & 0.16 \\
\hline TUM2 & 4 & 81.00 & 96.03 & 7.40 & 69.68 & 7.29 & 3.05 & 0.02 & 0.05 & 0.06 & 0.06 \\
\hline & \multicolumn{11}{|c|}{$C=10$} \\
\hline Dataset & $\sigma$ & Test & Train & $|w|_{1}$ & Time & \%Viol. & $e^{\top}(u+v)$ & $\% \mathrm{ft}(0)$ & $\% \mathrm{ft}(-2)$ & $\% \mathrm{ft}(-4)$ & $\% \mathrm{ft}(-9)$ \\
\hline $\mathrm{BC}$ & 63 & 93.17 & 92,48 & 1.36 & 0.59 & 0.29 & 1.32 & 2.00 & 34.00 & 34.00 & 34.00 \\
\hline PIMA & 17 & 75.57 & 76.19 & 5.89 & 3.80 & 0.0 & 3.50 & 26.25 & 43.75 & 43.75 & 43.75 \\
\hline HEART** & 17 & 83.95 & 86.92 & 7.28 & 0.1 & 29.35 & 6.95 & 13.85 & 99.23 & 100.00 & 100.00 \\
\hline IONO & 17 & 86.35 & 89,31 & 3.14 & 0.27 & 0.47 & 2.56 & 5.88 & 13.82 & 13.82 & 13.82 \\
\hline LIVER** & 6 & 74.89 & 74.39 & 5.77 & 0.02 & 10.19 & 3.93 & 64.00 & 100.00 & 100.00 & 100.00 \\
\hline
\end{tabular}

$S V M_{0}$ Algorithm - Tenfold cross validation 
We have run the algorithm on each dataset for different values of the penalty parameter $\sigma$, and we indicate in Table 4.5 the specific value of $\sigma$ the results refer to.

Some comments follow.

- In terms of classification correctness, the results of the $S V M_{0}$ Algorithm are comparable with those of $M B P$.

- The $S V M_{0}$ Algorithm provides better results in terms of number of zero-components of $w$. In fact, the percentage of components conventionally assumed equal to zero, that is $(100-\% f t(-9))$, is significantly bigger, except that in two cases, in $S V M_{0}$ Algorithm than in $M B P$.

- The computation time is, for both algorithms, negligible on the datasets of Group 2 while it is remarkably smaller for $S V M_{0}$ Algorithm as far as datasets of Group 1 are concerned.

In running the $S V M_{0}$ Algorithm, the most relevant issue is the appropriate tuning of the penalty parameter $\sigma>0$, which is, of course, dataset-specific. Two aspects are to be taken into account.

- "Small" values of $\sigma$ may lead to significant violation of the penalized constraint at the optimum of problem 3.20-3.23. Note that in such case, variables $u$ and $v$ may loose their "marker" role highlighted in Proposition 2.2.

- "Large" values of $\sigma$ may result in trivial solutions $(w=0)$ to the penalized problem (see Proposition 2.4). .

To illustrate in details the impact on the solution of parameter $\sigma$, we analyze the results for increasing values of $\sigma$. In particular we focus on the datasets CARC and IONO, from the first and second group, respectively. The results are reported in Tables 4.6 and 4.7 .

\begin{tabular}{|c|c|c|c|c|c|c|c|c|c|c|}
\hline \multicolumn{11}{|c|}{ Dataset : $C A R C ; C=1$} \\
\hline$\sigma$ & Test & Train & $\left.w\right|_{1}$ & Time & $\%$ Viol. & $e^{\top}(u+v)$ & $\% \mathrm{ft}(0)$ & $\% \mathrm{ft}(-2)$ & $\% \mathrm{ft}(-4)$ & $\% \mathrm{ft}(-9)$ \\
\hline 1 & 95.00 & 100,00 & 2.62 & 14.45 & 100 & 0.00 & 0.00 & 0.23 & 0.25 & 0.25 \\
\hline 2 & 94.17 & 100.00 & 3.50 & 73.13 & 44 & 0.87 & 0.01 & 0.14 & 0.15 & 0.15 \\
\hline 4 & 97.50 & 100,00 & 6.90 & 39,40 & 3 & 2.10 & 0.03 & 0.05 & 0.06 & 0.06 \\
\hline 5 & 91.67 & 100.00 & 6.26 & 41.41 & 1 & 2.07 & 0.02 & 0.03 & 0.03 & 0.03 \\
\hline 6 & 85.00 & 98.33 & 7.23 & 68.53 & 0.1 & 2.18 & 0.03 & 0.03 & 0.03 & 0.03 \\
\hline 7 & 75.83 & 96.99 & 6.08 & 93.80 & 0 & 2.20 & 0.03 & 0.03 & 0.03 & 0.03 \\
\hline 12 & 65.83 & 81,40 & 2.32 & 81.04 & 0 & 0.80 & 0.01 & 0.01 & 0.01 & 0.01 \\
\hline 16 & 58.33 & 57.59 & 0.0 & 1.81 & 0 & 0.0 & 0.0 & 0.0 & 0.0 & 0.0 \\
\hline
\end{tabular}

Results on dataset CARC for different values of $\sigma$

\begin{tabular}{|r|rrrrrrrrrr|}
\hline \multicolumn{10}{|c|}{ Dataset: $I O N O ; C=10$} \\
\hline$\sigma$ & Test & Train & $|w|_{1}$ & Time & \% Viol. $e^{\top}(u+v)$ & $\% \mathrm{ft}(0)$ & $\% \mathrm{ft}(-2)$ & $\% \mathrm{ft}(-4)$ & $\% \mathrm{ft}(-9)$ \\
\hline \hline 1 & 87.61 & 94.23 & 19.39 & 0.36 & 52 & 4.16 & 18.82 & 72.94 & 73.24 & 73.24 \\
2 & 87.94 & 93.53 & 11.21 & 0.22 & 50 & 4.34 & 9.41 & 62.06 & 63.53 & 63.53 \\
4 & 87.96 & 92.55 & 7.58 & 0.26 & 42 & 4.31 & 6.18 & 47.35 & 47.35 & 47.35 \\
10 & 87.63 & 89.38 & 4.23 & 0.24 & 19 & 2.90 & 5.88 & 18.53 & 19.41 & 19.41 \\
17 & 86.35 & 89.31 & 3.14 & 0.29 & 15 & 2.56 & 5.88 & 13.82 & 13.82 & 13.82 \\
30 & 80.80 & 83.47 & 1.83 & 0.23 & 6 & 1.77 & 3.82 & 7.65 & 7.94 & 7.04 \\
50 & 74.77 & 74.68 & 1.00 & 0.26 & 3 & 0.97 & 2.94 & 2.94 & 2.94 & 2.94 \\
64 & 64.33 & 64.24 & 0.0001 & 1.84 & 0.0 & 0.0001 & 0.0 & 0.0 & 0.29 & 0.29 \\
93 & 64.33 & 64.24 & 0.0 & 0.16 & 0 & 0.0 & 0.0 & 0.0 & 0.0 & 0.0 \\
\hline
\end{tabular}

Results on dataset IONO for different values of $\sigma$

\footnotetext{
${ }^{\ddagger}$ Symbols "** and "**" in Dataset column of Table 4.5 indicate that parameter $C$ has been set to 2 and 100 , respectively.
} 
We observe that on both datasets increasing values of $\sigma$ results, as expected, in deterioration of the classification correctness (under such point of view, significant data are those obtained in the training phase). On the other hand, the violation of the relaxed constraint gets smaller and smaller, until, for too large values of $\sigma$, the results become meaningless, as $w$ gets close to zero.

5. Conclusions. We have tackled the Feature Selection problem within SVM binary classification by using the polyhedral $k$-norm, in the sparse optimization context. The numerical experiments show that the approach is a promising alternative to continuous approximations of the $\ell_{0}$ pseudo-norm and to integer programming-based methods.

\section{REFERENCES}

[1] E. Amaldi, V. Kann, On the approximability of minimizing nonzero variables or unsatisfied relations in linear systems, Theoretical Computer Science, 1998, 209, 12, pp. 237-260.

[2] P. Bertolazzi, G. Felici, P. Festa, G. Fiscon, E. Weitschek, Integer programming models for feature selection: New extensions and a randomized solution algorithm, European Journal of Operational Research, 2016, 250, 2, pp. 389-399.

[3] P.S. Bradley, O.L. Mangasarian, W.N. Street, Feature selection via mathematical programming, INFORMS Journal on Computing, 1998, 10, 2, pp. 209-217.

[4] N. Cristianini, J. Shawe-Taylor, 2000 . An introduction to support vector machines and other kernel-based learning methods, 2000, Cambridge University Press.

[5] G. Di Pillo, L. Grippo, Exact penalty functions in constrained optimization, SIAM Journal on Control and Optimization, 1989, 27, 6, pp. 1333-1360.

[6] G. Gasso, A. Rakotomamonjy, S. Canu, Recovering Sparse Signals With a Certain Family of Nonconvex Penalties and DC Programming, IEEE Transactions on Signal Processing, 2009, 57, 12, pp. 4686-4698.

[7] M. Gaudioso, E. Gorgone, M. Labbé, A. M. Rodríguez-Chía, Lagrangian relaxation for SVM feature selection, Computers and Operations Research 2017, 87, pp. 137-145.

[8] M. Gaudioso, G. Giallombardo, G. Miglionico, A. M. Bagirov, Minimizing nonsmooth DC functions via successive DC piecewise-affine approximations, Journal of Global Optimization, 2018, 71, 1, pp.37-55.

[9] J.Gotoh, A. Takeda, K.Tono, DC formulations and algorithms for sparse optimization problems, Mathematical Programming, Ser. B, 2018, 169, 1, pp. 141-176.

[10] I. Guyon, A. Elisseeff, 2003. An introduction to variable and feature selection, Journal of Machine Learning Research, 2003, 3, pp. 11571182.

[11] A. B. Hempel, P. J. Goulart, A Novel Method for Modelling Cardinality and Rank Constraints, 53rd IEEE Conference on Decision and Control, Los Angeles, Cal., USA Dec. 15-17, 2014, pp. 4322-4327.

[12] J.-B. Hiriart-Urruty, Generalized differentiability/ duality and optimization for problems dealing with differences of convex functions, Lecture Notes in Economic and Mathematical Systems, 1986, 256, Springer Verlag, pp. 37-70.

[13] J.-B. Hiriart-Urruty, D. Ye, Sensitivity analysis of all eigevalues of a symmetric matrix, Numerische Mathematik, 1995, 70, 1, pp. 45-72.

[14] K. Joki, A.M. Bagirov, N. Karmitsa, and M.M. Mäkelä, A proximal bundle method for nonsmooth DC optimization utilizing nonconvex cutting planes, Journal of Global Optimization, 2017, 68, 3, pp. 501-535. -

[15] H.A. Le Thi, T. Pham Dinh, The DC (difference of convex functions) programming and DCA revisited with DC models of real world nonconvex optimization problems, Journal of Global Optimization, 2005, 133, pp. 23-46.

[16] H.A. Le Thi, H.M. Le, V.V. Nguyen, T. Pham Dinh, A DC programming approach for feature selection in support vector machines learning, Advances in Data Analysis and Classification, 2008, 2, pp. 259278.

[17] S. Maldonado, J. Pérez, R. Weber, M. Labbé, Feature selection for Support Vector Machines via Mixed Integer Linear Programming, Information Sciences, 2014, 279, pp. 163-175.

[18] O.L. Mangasarian, Nonlinear Programming, 1969, McGraw-Hill.

[19] M.L. Overton, R.S. Womersley, Optimality conditions and duality theory for minimizing sums of the largest eigenvalues of symmetric matrices, Mathematical Programming, 1993, 62, 1-3, pp. 321-357.

[20] M. Pilanci, M. J. Wainwright, L. El Ghaoui, Sparse learning via Boolean relaxations, Mathematical Programming, Ser. B, 2015, 151, pp. 63-87.

[21] F. Rinaldi, F. Schoen, M. Sciandrone, Concave programming for minimizing the zero-norm over polyhedral sets, Computational Optimization and Applications, 2010, 46, pp. 467486. 
[22] A.S. Strekalovsky, Global optimality conditions for nonconvex optimization, Journal of Global Optimization, 1998, 12 , pp. 415-434.

[23] E. Soubies, L. Blanc-Féraud, G. Aubert, A Unified View of Exact Continuous Penalties for $\ell_{2}-\ell_{0}$ Minimization, SIAM Journal on Optimization, 2017, 27, 3, pp. 2034-2060.

[24] V. Vapnik, The nature of the statistical learning theory, 1995, Springer Verlag.

[25] G.A. Watson, Linear best approximation using a class of polyhedral norms, Numerical Algorithms, 1992, 2 pp. 321-336.

[26] J. Weston, A. Elisseeff, B. Schölkopf, M. Tipping, Use of the zero-norm with linear models and kernel methods, Journal of Machine Learning Research, 2003, 3, pp. 1439-1461.

[27] S. J. Wright, Accelerated block-cordinate relaxation for regularized optimization, SIAM Journal on Optimization, 2012, 22, 1, pp. 159-186.

[28] B. Wu, C. Ding, D. Sun, K-C. Toh, On the Moreau-Yosida regularization of the vector $k-$ norm related functions, SIAM Journal on Optimization, 2014, 24. 2, pp. 766-794. 